\title{
Properties of the linear canonical integral transformation
}

\author{
Tatiana Alieva ${ }^{1}$ and Martin J. Bastiaans ${ }^{2}$ \\ ${ }^{1}$ Universidad Complutense de Madrid, Facultad de Ciencias Físicas, Ciudad Universitaria s/n, Madrid 28040, Spain \\ ${ }^{2}$ Technische Universiteit Eindhoven, Faculteit Elektrotechniek, Postbus 513, 5600 MB Eindhoven, Netherlands \\ *Corresponding author: m.j.bastiaans@tue.nl
}

Received May 4, 2007; revised September 19, 2007; accepted October 2, 2007; posted October 3, 2007 (Doc. ID 82777); published October 30, 2007

We provide a general expression and different classification schemes for the general two-dimensional canonical integral transformations that describe the propagation of coherent light through lossless first-order optical systems. Main theorems for these transformations, such as shift, scaling, derivation, etc., together with the canonical integral transforms of selected functions, are derived. (C) 2007 Optical Society of America

OCIS codes: $070.2580,070.4690,070.6760,080.2730,110.6980,120.4820,350.6980$.

\section{INTRODUCTION}

Linear canonical integral transformations [1-4] (CTs) play an important role in optical and digital information processing: They produce affine signal transformations in phase space. During the past decade, many new signal processing operations have entered the realm of traditional Fourier optics. Some of them belong to the class of the CTs, as is the case for the fractional Fourier transformation [5], and others-such as the wavelet transformation, fractional convolution, and the Wigner distribution-can be represented as linear combinations of CTs or as cascades of such transformations with intermediate multiplicative displays that can be realized by spatial light modulators [6]. There exists a long list of papers where the properties and applications of twodimensional CTs have been studied [1-5]; we refer to these papers and to the references therein. Unfortunately, most of them restrict themselves to the case of symmetrical or separable CTs only, whereas nonseparable CTs are the ones that actually open new perspectives for information processing. Moreover, such nonseparable CTs are able to describe optical systems that serve as mode converters $[7,8]$ and as nonconventional optical resonators.

The purpose of this paper is to provide an expression for the general (nonseparable) two-dimensional CT and to present its main properties, including the shift, scaling, and derivation theorems, well known for the Fourier transformation. Based on the ray transformation matrix formalism, we also propose a classification of these CTs. Finally, the CTs of some selected functions are derived.

\section{CANONICAL INTEGRAL TRANSFORMATION}

\section{A. Definition}

The linear canonical integral transformation describes-in the paraxial approximation of the scalar diffraction theory-the evolution of the complex field amplitude $f(\mathbf{r})$ during its propagation through a first-order optical system:

$$
f_{i}\left(\mathbf{r}_{i}\right) \rightarrow f_{o}\left(\mathbf{r}_{o}\right)=\mathcal{R}^{\mathbf{T}}\left[f_{i}\left(\mathbf{r}_{i}\right)\right]\left(\mathbf{r}_{o}\right) .
$$

In what follows, we will often use the shorthand notation $\mathcal{R}^{\mathbf{T}}\left[f\left(\mathbf{r}_{i}\right)\right]\left(\mathbf{r}_{o}\right)=F_{\mathbf{T}}\left(\mathbf{r}_{o}\right)$. The CT kernel of the operator $\mathcal{R}^{\mathbf{T}}$ is parameterized by the system's well-known $4 \times 4$ real symplectic ray transformation matrix $\mathbf{T}$, which relates the position $\mathbf{r}_{i}$ and direction $\mathbf{q}_{i}$ of an incoming ray to the position $\mathbf{r}_{o}$ and direction $\mathbf{q}_{o}$ of the outgoing ray:

$$
\left(\begin{array}{l}
\mathbf{r}_{o} \\
\mathbf{q}_{o}
\end{array}\right)=\left[\begin{array}{ll}
\mathbf{A} & \mathbf{B} \\
\mathbf{C} & \mathbf{D}
\end{array}\right]\left(\begin{array}{l}
\mathbf{r}_{i} \\
\mathbf{q}_{i}
\end{array}\right)=\mathbf{T}\left(\begin{array}{l}
\mathbf{r}_{i} \\
\mathbf{q}_{i}
\end{array}\right),
$$

where $\mathbf{r}=(x, y)^{t}$ denote (dimensionless) spatial variables and $\mathbf{q}=\left(q_{x}, q_{y}\right)^{t}$ are their conjugates: the (also dimensionless) spatial-frequency (or direction) variables. As usual, the denotes transposition. While lower-case boldface letters will throughout denote two-dimensional vectors, upper-case boldface letters will indicate matrices, where $\mathbf{T}$ and $\mathbf{M}$ are used for $4 \times 4$ matrices and the other letters for $2 \times 2$ ones. The inverse transformation is parameterized by the matrix $\mathbf{T}^{-1}$.

Since the ray transformation matrix $\mathbf{T}$ is symplectic,

$$
\left[\begin{array}{ll}
\mathbf{A} & \mathbf{B} \\
\mathbf{C} & \mathbf{D}
\end{array}\right]^{-1}=\left[\begin{array}{cc}
\mathbf{D}^{t} & -\mathbf{B}^{t} \\
-\mathbf{C}^{t} & \mathbf{A}^{t}
\end{array}\right],
$$

and thus

$$
\begin{aligned}
& \mathbf{A B}^{t}=\mathbf{B A}^{t}, \quad \mathbf{C D}^{t}=\mathbf{D C}^{t}, \quad \mathbf{A D}^{t}-\mathbf{B C}^{t}=\mathbf{I} ; \\
& \mathbf{A}^{t} \mathbf{C}=\mathbf{C}^{t} \mathbf{A}, \quad \mathbf{B}^{t} \mathbf{D}=\mathbf{D}^{t} \mathbf{B}, \quad \mathbf{A}^{t} \mathbf{D}-\mathbf{C}^{t} \mathbf{B}=\mathbf{I} ;
\end{aligned}
$$

with I throughout denoting the identity matrix; it has only ten free parameters in the two-dimensional case that we consider in this paper. Moreover, any symplectic matrix can be decomposed in the modified Iwasawa form [9] as 


$$
\left[\begin{array}{ll}
\mathbf{A} & \mathbf{B} \\
\mathbf{C} & \mathbf{D}
\end{array}\right]=\left[\begin{array}{cc}
\mathbf{I} & \mathbf{0} \\
\mathbf{- G} & \mathbf{I}
\end{array}\right]\left[\begin{array}{cc}
\mathbf{S} & \mathbf{0} \\
\mathbf{0} & \mathbf{S}^{-1}
\end{array}\right]\left[\begin{array}{cc}
\mathbf{X} & \mathbf{Y} \\
-\mathbf{Y} & \mathbf{X}
\end{array}\right]
$$

in which the first matrix on the right-hand side represents a generalized lens described by the symmetric matrix

$$
\mathbf{G}=-\left(\mathbf{C A}^{t}+\mathbf{D B}^{t}\right)\left(\mathbf{A A}^{t}+\mathbf{B B}^{t}\right)^{-1}=\mathbf{G}^{t},
$$

the second matrix corresponds to a magnifier described by the positive definite symmetric matrix

$$
\mathbf{S}=\left(\mathbf{A A}^{t}+\mathbf{B B}^{t}\right)^{1 / 2}=\mathbf{S}^{t},
$$

and the third is an orthosymplectic [10] (i.e., both orthogonal and symplectic) matrix that can be represented elegantly by the unitary matrix

$$
\mathbf{U}=\mathbf{X}+i \mathbf{Y}=\left(\mathbf{A A}^{t}+\mathbf{B B}^{t}\right)^{-1 / 2}(\mathbf{A}+i \mathbf{B}) .
$$

In the often used case $\operatorname{det} \mathbf{B} \neq 0$, the $\mathrm{CT}$ takes the form of Collins integral [11]:

$$
\begin{aligned}
f_{o}\left(\mathbf{r}_{o}\right)= & \mathcal{R}^{\mathbf{T}}\left[f_{i}\left(\mathbf{r}_{i}\right)\right]\left(\mathbf{r}_{o}\right)=(\operatorname{det} i \mathbf{B})^{-1 / 2} \int_{-\infty}^{\infty} f_{i}\left(\mathbf{r}_{i}\right) \\
& \times \exp \left[i \pi\left(\mathbf{r}_{i}^{t} \mathbf{B}^{-1} \mathbf{A} \mathbf{r}_{i}-2 \mathbf{r}_{i}^{t} \mathbf{B}^{-1} \mathbf{r}_{o}+\mathbf{r}_{o}^{t} \mathbf{D} \mathbf{B}^{-1} \mathbf{r}_{o}\right)\right] \mathrm{d} \mathbf{r}_{i} .
\end{aligned}
$$

Note that if $\mathbf{A}=\mathbf{D}^{\mathrm{t}}$ and $\mathbf{B}=\mathbf{B}^{\mathrm{t}}$ (and hence also $\mathbf{C}=\mathbf{C}^{\mathrm{t}}$ ), the integral kernel is symmetric in the sense that we may interchange $\mathbf{r}_{o}$ and $\mathbf{r}_{i}$. The ray-reversed system, whose ray transformation matrix $\hat{\mathbf{T}}$ takes the form

$$
\hat{\mathbf{T}}=\left[\begin{array}{cc}
\mathbf{A} & -\mathbf{B} \\
-\mathbf{C} & \mathbf{D}
\end{array}\right]^{-1}
$$

is then identical to the initial one and we have indeed $\hat{\mathbf{T}}$ $=\mathbf{T}$.

A simple expression can also be written in the case $\mathbf{B}$ $=\mathbf{0}$, which corresponds to the generalized imaging condition, including a possible scaling and rotation of the input function accompanied by an additional phase modulation:

$$
f_{o}(\mathbf{r})=(|\operatorname{det} \mathbf{A}|)^{-1 / 2} \exp \left(i \pi \mathbf{r}^{t} \mathbf{C A}^{-1} \mathbf{r}\right) f_{i}\left(\mathbf{A}^{-1} \mathbf{r}\right) .
$$

To deal with the singular case $\operatorname{det} \mathbf{B}=0$, but $\mathbf{B} \neq \mathbf{0}$, we can use the Iwasawa decomposition (3), from which we note that $\mathbf{B}=\mathbf{S Y}$. Since the scaling matrix $\mathbf{S}$ is nonsingular, a singularity of $\mathbf{B}$ is due only to the orthosymplectic part (6) of the Iwasawa decomposition. We will return to this case at the end of this section, after having studied in more detail the optical realization of an orthosymplectic system as a separable fractional Fourier transformer embedded between two rotators [12].

In information processing a signal is often described by its Fourier transform (FT), which corresponds in coherent optics to the angular spectrum, i.e., the FT $\mathcal{F}[f(\mathbf{r})](\mathbf{q})$ of the complex field amplitude $f(\mathbf{r})$ :

$$
\mathcal{F}[f(\mathbf{r})](\mathbf{q})=\int_{-\infty}^{\infty} f(\mathbf{r}) \exp \left(-i 2 \pi \mathbf{q}^{t} \mathbf{r}\right) \mathrm{d} \mathbf{r} .
$$

If a complex field amplitude is canonically transformed with the matrix $\mathbf{T}$, its angular spectrum is canonically transformed with $\left(\mathbf{T}^{t}\right)^{-1}$ :

$$
\left(\mathbf{T}^{t}\right)^{-1}=\left[\begin{array}{cc}
\mathbf{D} & -\mathbf{C} \\
-\mathbf{B} & \mathbf{A}
\end{array}\right]
$$

We finally remark the intimate relation between the CT and the description of a signal $f(\mathbf{r})$ by means of its Wigner distribution

$$
W_{f}(\mathbf{r}, \mathbf{q})=\int_{-\infty}^{\infty} f\left(\mathbf{r}+\mathbf{r}^{\prime} / 2\right) f^{*}\left(\mathbf{r}-\mathbf{r}^{\prime} / 2\right) \exp \left(-i 2 \pi \mathbf{q}^{t} \mathbf{r}^{\prime}\right) \mathrm{d} \mathbf{r}^{\prime} .
$$

In terms of the Wigner distribution, the CT takes the elegant form

$$
W_{f}(\mathbf{r}, \mathbf{q})=W_{\mathcal{R}^{\mathbf{T}}[f(\cdot)]}(\mathbf{A r}+\mathbf{B q}, \mathbf{C r}+\mathbf{D q}),
$$

which holds independently of the regularity of the matrix B (which was the condition for the Collins integral), and we conclude that the CT merely causes an affine transformation of the coordinates $(\mathbf{r}, \mathbf{q})$ in phase space. A similar relation holds for the signal's ambiguity function

$$
A_{f}\left(\mathbf{r}^{\prime}, \mathbf{q}^{\prime}\right)=\int_{-\infty}^{\infty} f\left(\mathbf{r}+\mathbf{r}^{\prime} / 2\right) f^{*}\left(\mathbf{r}-\mathbf{r}^{\prime} / 2\right) \exp \left(-i 2 \pi \mathbf{r}^{t} \mathbf{q}^{\prime}\right) \mathrm{d} \mathbf{r}
$$

for which we have, in analogy with Eq. (12),

$$
A_{f}\left(\mathbf{r}^{\prime}, \mathbf{q}^{\prime}\right)=A_{\mathcal{R}^{\mathbf{T}}[f(\cdot)]}\left(\mathbf{A} \mathbf{r}^{\prime}+\mathbf{B} \mathbf{q}^{\prime}, \mathbf{C} \mathbf{r}^{\prime}+\mathbf{D} \mathbf{q}^{\prime}\right)
$$

\section{B. Classification}

To understand the CTs' actions, they can be classified based on the values of the submatrices $\mathbf{A}, \mathbf{B}, \mathbf{C}$, and $\mathbf{D}$. Let us first consider the situation where one or two of the submatrices are zero; note that the case $\mathbf{B}=\mathbf{0}$ has already been mentioned; see Eq. (8).

If $\mathbf{A}=\mathbf{0}$ (and consequently $\mathbf{C}^{t}=-\mathbf{B}^{-1}$ ), we obtain that the transformation (7) describes a kind of Fourier transforming system that-besides the FT-produces also an appropriate scaling, rotation, and phase modulation. The phase modulation vanishes for $\mathbf{D = 0}$. A pure $\mathrm{FT}$ (without any additional scaling, rotation, and phase modulation) arises when $\mathbf{A}=\mathbf{D}=\mathbf{0}$ and $\mathbf{B}=-\mathbf{C}=\mathbf{I}$.

The case $\mathbf{D}=\mathbf{0}$ (and again $\mathbf{C}^{t}=-\mathbf{B}^{-1}$ ) corresponds to a similar FT-like transformation, but now with respect to the angular spectrum, in agreement with Eq. (10).

The case $\mathbf{C}=\mathbf{0}$ (and thus $\mathbf{D}^{t}=\mathbf{A}^{-1}$ ) can be considered as a generalized Fresnel transformation or convolution-type transformation, and corresponds to a Fouriertransformed version of Eq. (8); indeed, using Eq. (10) we get

$$
\begin{aligned}
\mathcal{F}\left[f_{o}(\mathbf{r})\right](\mathbf{q}) & \left.=(|\operatorname{det} \mathbf{D}|)^{-1 / 2} \exp \left(-i \pi \mathbf{q}^{t} \mathbf{B D}^{-1} \mathbf{q}\right) \mathcal{F} f_{i}(\mathbf{r})\right]\left(\mathbf{D}^{-1} \mathbf{q}\right) \\
& =(|\operatorname{det} \mathbf{A}|)^{1 / 2} \exp \left(-i \pi \mathbf{q}^{t} \mathbf{B} \mathbf{A}^{t} \mathbf{q}\right) \mathcal{F}\left[f_{i}(\mathbf{r})\right]\left(\mathbf{A}^{t} \mathbf{q}\right)
\end{aligned}
$$

The evolution of the Fourier spectrum corresponds to an affine transformation determined by the matrix $\mathbf{D}^{-1}=\mathbf{A}^{t}$, together with an additional phase modulation, which resembles what happens with the spectrum under Fresnel transformation. 
Another classification scheme is based on the kernel symmetry. If all submatrices are scalar, $\mathbf{A}=a \mathbf{I}, \mathbf{B}=b \mathbf{I}, \mathbf{C}$ $=c \mathbf{I}$, and $\mathbf{D}=d \mathbf{I}$, then the CT is isotropic and is described by only three free parameters. This is the case, for example, for the Fresnel transformation described by the parameter $b$ and with $a=d=1$ and $c=0$, and for the symmetric fractional FT described by the parameter $\gamma$ and with $a=d=\cos \gamma$ and $b=-c=\sin \gamma$.

If the matrices $\mathbf{A}, \mathbf{B}, \mathbf{C}$, and $\mathbf{D}$ are diagonal, we obtain separable CTs with the isotropic CTs as a particular case. Separable CTs describe first-order optical systems composed of sections of free space and sets of cylindrical lenses that are parallel with or perpendicular to the chosen coordinate axes (with spherical lenses as a particular case). Separable CTs have six free parameters. As an example, we mention the separable fractional FT $\mathbf{T}_{f}\left(\gamma_{x}, \gamma_{y}\right)$ described by the matrices

$$
\begin{gathered}
\mathbf{A}_{f}=\mathbf{D}_{f}=\left[\begin{array}{cc}
\cos \gamma_{x} & 0 \\
0 & \cos \gamma_{y}
\end{array}\right] \equiv \mathbf{X}_{f}\left(\gamma_{x}, \gamma_{y}\right), \\
\mathbf{B}_{f}=-\mathbf{C}_{f}=\left[\begin{array}{cc}
\sin \gamma_{x} & 0 \\
0 & \sin \gamma_{y}
\end{array}\right] \equiv \mathbf{Y}_{f}\left(\gamma_{x}, \gamma_{y}\right),
\end{gathered}
$$

which corresponds to rotations in the $\left(x, q_{x}\right)$ and $\left(y, q_{y}\right)$ planes through the angles $\gamma_{x}$ and $\gamma_{y}$, respectively. Note that $\mathcal{R}^{\mathbf{T}_{f}(\pi / 2, \pi / 2)}[f(\mathbf{r})](\mathbf{q})$ corresponds to the common FT $\mathcal{F}[f(\mathbf{r})](\mathbf{q})$, apart from a constant phase factor $\exp (-i \pi / 2)$.

To permit rotations in other planes of the phase space, we need nonseparable (general) CTs. Then such operations as rotation, gyration, and skew become possible. Thus, the rotator operation $\mathbf{T}_{r}(\alpha)$ with

$$
\begin{gathered}
\mathbf{A}_{r}=\mathbf{D}_{r}=\left[\begin{array}{cc}
\cos \alpha & \sin \alpha \\
-\sin \alpha & \cos \alpha
\end{array}\right] \equiv \mathbf{X}_{r}(\alpha), \\
\mathbf{B}_{r}=\mathbf{C}_{r}=\mathbf{0} \equiv \mathbf{Y}_{r}(\alpha),
\end{gathered}
$$

produces a rotation of the image and its FT (the angular spectrum) through an angle $\alpha$. The gyrator operation $\mathbf{T}_{g}(\vartheta)$ with

$$
\begin{gathered}
\mathbf{A}_{g}=\mathbf{D}_{g}=\left[\begin{array}{cc}
\cos \vartheta & 0 \\
0 & \cos \vartheta
\end{array}\right] \equiv \mathbf{X}_{g}(\vartheta), \\
\mathbf{B}_{g}=-\mathbf{C}_{g}=\left[\begin{array}{cc}
0 & \sin \vartheta \\
\sin \vartheta & 0
\end{array}\right] \equiv \mathbf{Y}_{g}(\vartheta),
\end{gathered}
$$

corresponds to twisting, i.e., rotations in the $\left(x, q_{y}\right)$ and $\left(y, q_{x}\right)$ planes. Optical systems that perform these two transforms and that are composed of generalized lenses have been proposed in [13].

Note that the matrices described by Eqs. (16)-(18) are orthosymplectic, and the related systems can therefore be easily described by unitary matrices $\mathbf{U}=\mathbf{X}+i \mathbf{Y}$, as introduced in Eq. (6).

While the Fresnel and lens transformations correspond to shearing in the $\left(x, q_{x}\right)$ and $\left(y, q_{y}\right)$ planes [14], the skew operation with

$$
\mathbf{A}_{s}=\left[\begin{array}{ll}
1 & u \\
0 & 1
\end{array}\right], \quad \mathbf{D}_{s}=\left[\begin{array}{cc}
1 & 0 \\
-u & 1
\end{array}\right], \quad \mathbf{B}_{s}=\mathbf{C}_{s}=\mathbf{0}
$$

is related to shearing operations in the $(x, y)$ and $\left(q_{x}, q_{y}\right)$ planes. Using, for example, the Iwasawa decomposition, Eq. (3), we can show that such a shearing operation in the position (and frequency) plane can be performed by a combination of scaling described by the symmetric matrix

$$
\mathbf{S}_{s}=\left[\begin{array}{cc}
\left(1+\sin ^{2} \theta\right) / \cos \theta & \sin \theta \\
\sin \theta & \cos \theta
\end{array}\right]
$$

and a rotation through the angle $\theta$ described by the ma$\operatorname{trix} \mathbf{X}_{r}(\theta)$, where $2 \tan \theta=u$. Indeed, $\mathbf{A}_{s}=\mathbf{S}_{s} \mathbf{X}_{r}(\theta)$.

A general CT has ten free parameters and can be performed optically by application of cylindrical lenses oriented at the appropriate angles with respect to the transverse axes [15].

A third classification scheme for CTs based on the analysis of the eigenvalues of the ray transformation matrix is given in [16]. This scheme is important for the determination of the eigenfunctions of the CT and for the stability analysis of resonators. Expressions for the eigenfunctions of a CT for systems with unimodular eigenvalues can be found in $[17,18]$, while explicit expressions in the case of real-valued eigenvalues have been derived in [19].

\section{General Expression}

We are now prepared to consider again the case of a possible singularity of the submatrix $\mathbf{B}$. We first recall that any orthosymplectic matrix can be decomposed in the form [12] $\mathbf{T}_{r}(\beta) \mathbf{T}_{f}\left(\gamma_{x}, \gamma_{y}\right) \mathbf{T}_{r}(\alpha)$, where a separable fractional Fourier transformer is embedded between two rotators. When we substitute this cascade into the Iwasawa decomposition (3), we are led to a cascade with a clear physical interpretation. The cascade starts with a rotator $\mathbf{T}_{r}(\alpha)$ that rotates the coordinate system such that the new axes coincide with the axes of the separable fractional Fourier transformer $\mathbf{T}_{f}\left(\gamma_{x}, \gamma_{y}\right)$. This separable fractional Fourier transformer is responsible for a possible singularity of the submatrix $\mathbf{B}$, but such a singularity now has a clear interpretation: it simply means that for one coordinate (or maybe even for both coordinates) the fractional Fourier transformer acts as an identity system, i.e., $\gamma_{x}=0$ and $/$ or $\gamma_{y}=0$. The cascade then continues with the rotator $\mathbf{T}_{r}(\beta)$, followed by the magnifier (with matrix $\mathbf{S}$ ) and the lens (with matrix $\mathbf{G}$ ), and brings us to the final input-output relationship for the entire cascade [12]:

$$
\begin{aligned}
f_{o}\left(\mathbf{r}_{o}\right)= & (\operatorname{det} \mathbf{S})^{-1 / 2} \exp \left(-i \pi \mathbf{r}_{o}^{t} \mathbf{G} \mathbf{r}_{o}\right) \\
& \times \mathcal{R}^{\mathbf{T}_{f}\left(\gamma_{x}, \gamma_{y}\right)}\left[f_{i}\left(\mathbf{X}_{r}(\alpha) \mathbf{r}_{i}\right)\right]\left[\mathbf{X}_{r}(-\beta) \mathbf{S}^{-1} \mathbf{r}_{o}\right]
\end{aligned}
$$

Equation (21) provides a general representation of the linear canonical integral transformation, which is valid for any ray transformation matrix, with or without a singularity of the submatrix $\mathbf{B}$. 


\section{MAIN THEOREMS FOR THE CANONICAL INTEGRAL TRANSFORMATION}

The $\mathrm{CT}$ is a linear transform: $\mathcal{R}^{\mathbf{T}}[f(\cdot)+g(\cdot)](\mathbf{r})$ $=\mathcal{R}^{\mathbf{T}}[f(\cdot)](\mathbf{r})+\mathcal{R}^{\mathbf{T}}[g(\cdot)](\mathbf{r})$. The cascade of two CTs associated with the ray transformation matrices $\mathbf{T}_{1}$ and $\mathbf{T}_{2}$ is a CT parameterized by the matrix product

$$
\mathbf{T}_{2} \mathbf{T}_{1}: \mathcal{R}^{\mathbf{T}_{2}}\left[\mathcal{R}^{\mathbf{T}_{1}}[f(\cdot)](\cdot)\right](\mathbf{r})=\mathcal{R}^{\mathbf{T}_{2} \mathbf{T}_{1}}[f(\cdot)](\mathbf{r}) .
$$

Then the inverse CT to the CT $\mathcal{R}^{\mathbf{T}}$ is described by the matrix $\mathbf{T}^{-1}$, as we mentioned before.

We also mention the property

$$
\left\{\mathcal{R}^{\mathbf{T}}[f(\cdot)](\mathbf{r})\right\}^{*}=\mathcal{R}^{\hat{\mathbf{T}}^{-1}}\left[f^{*}(\cdot)\right](\mathbf{r}),
$$

from which we conclude that the complex conjugated version of the CT of a certain function $f$ is equal to the result that we get when the inverse of the ray-reversed system operates on the complex conjugated version $f^{*}$ of that function, and the well-known Parseval theorem

$$
\int_{-\infty}^{\infty} f\left(\mathbf{r}_{i}\right) g^{*}\left(\mathbf{r}_{i}\right) \mathrm{d} \mathbf{r}_{i}=\int_{-\infty}^{\infty} \mathcal{R}^{\mathbf{T}}\left[f\left(\mathbf{r}_{i}\right)\right]\left(\mathbf{r}_{o}\right)\left\{\mathcal{R}^{\mathbf{T}}\left[g\left(\mathbf{r}_{i}\right)\right]\left(\mathbf{r}_{o}\right)\right\}^{*} \mathrm{~d} \mathbf{r}_{o}
$$

for lossless transformations, which in particular yields the energy preservation law

$$
\int_{-\infty}^{\infty}\left|f\left(\mathbf{r}_{i}\right)\right|^{2} \mathrm{~d} \mathbf{r}_{i}=\int_{-\infty}^{\infty}\left|\mathcal{R}^{\mathbf{T}}\left[f\left(\mathbf{r}_{i}\right)\right]\left(\mathbf{r}_{o}\right)\right|^{2} \mathrm{~d} \mathbf{r}_{o} .
$$

The application of the CT for optical information processing requires the generalization of the well-known theorems for the FT, such as shift, scaling, derivation, etc., to the case of the CT.

\section{A. Shift Theorem}

A shift of the input field with respect to the optical axis by a vector $\mathbf{v}, f_{i}(\mathbf{r}) \rightarrow f_{i}(\mathbf{r}-\mathbf{v})$, leads to a shift of the output signal by the vector Av and to an additional quadratic phase factor; in detail we have

$$
\mathcal{R}^{\mathbf{T}}\left[f_{i}\left(\mathbf{r}_{i}-\mathbf{v}\right)\right]\left(\mathbf{r}_{o}\right)=\exp \left[i \pi\left(2 \mathbf{r}_{o}-\mathbf{A v}\right)^{t} \mathbf{C v}\right] \mathcal{R}^{\mathbf{T}}\left[f_{i}\left(\mathbf{r}_{i}\right)\right]\left(\mathbf{r}_{o}-\mathbf{A v}\right),
$$

where we have used the symplecticity conditions (2) and the fact that $\mathbf{v}^{t} \mathbf{Z q}=\mathbf{q}^{t} \mathbf{Z}^{t} \mathbf{v}$. The shift theorem (25) implies that the intensity distribution does not change due to a displacement by $\mathbf{v}$, but is merely shifted by Av:

$$
\left|\mathcal{R}^{\mathbf{T}}\left[f\left(\mathbf{r}_{i}-\mathbf{v}\right)\right]\left(\mathbf{r}_{o}\right)\right|=\left|F_{\mathbf{T}}\left(\mathbf{r}_{o}-\mathbf{A v}\right)\right| .
$$

In particular, Eq. (25) reduces to

$$
\mathcal{R}^{\mathbf{T}}\left[f\left(\mathbf{r}_{i}-\mathbf{v}\right)\right]\left(\mathbf{r}_{o}\right)=F_{\mathbf{T}}\left(\mathbf{r}_{o}-\mathbf{A v}\right)
$$

for $\mathbf{C}=\mathbf{0}$, and for $\mathbf{A}=\mathbf{0}$ it reduces to

$$
\mathcal{R}^{\mathbf{T}}\left[f\left(\mathbf{r}_{i}-\mathbf{v}\right)\right]\left(\mathbf{r}_{o}\right)=\exp \left(i \pi 2 \mathbf{r}_{o}^{t} \mathbf{C v}\right) \boldsymbol{F}_{\mathbf{T}}\left(\mathbf{r}_{o}\right) .
$$

We again stress the similarity of the CT to the Fresnel transformation and the FT for the cases $\mathbf{C}=\mathbf{0}$ and $\mathbf{A}=\mathbf{0}$, respectively.

\section{B. Convolution Theorem}

Using the shift theorem, the CT of the convolution operation

$$
(f * h)(\mathbf{r})=\int_{-\infty}^{\infty} f(\mathbf{r}-\mathbf{v}) h(\mathbf{v}) \mathrm{d} \mathbf{v}=\int_{-\infty}^{\infty} h(\mathbf{r}-\mathbf{v}) f(\mathbf{v}) \mathrm{d} \mathbf{v}
$$

can be written in the form

$$
\begin{aligned}
\mathcal{R}^{\mathbf{T}}\left[(f * h)\left(\mathbf{r}_{i}\right)\right]\left(\mathbf{r}_{o}\right) \\
\quad=\int_{-\infty}^{\infty} \exp \left[i \pi\left(2 \mathbf{r}_{o}-\mathbf{A v}\right)^{t} \mathbf{C v}\right] F_{\mathbf{T}}\left(\mathbf{r}_{o}-\mathbf{A v}\right) h(\mathbf{v}) \mathrm{d} \mathbf{v} \\
\quad=\int_{-\infty}^{\infty} \exp \left[i \pi\left(2 \mathbf{r}_{o}-\mathbf{A v}\right)^{t} \mathbf{C v}\right] H_{\mathbf{T}}\left(\mathbf{r}_{o}-\mathbf{A v}\right) f(\mathbf{v}) \mathrm{d} \mathbf{v} .
\end{aligned}
$$

In the case $\mathbf{A}=\mathbf{0}$ (and thus also $\mathbf{C}^{t}=-\mathbf{B}^{-1}$ ), we get

$$
\begin{aligned}
\mathcal{R}^{\mathbf{T}}\left[(f * h)\left(\mathbf{r}_{i}\right)\right]\left(\mathbf{r}_{o}\right)= & (\operatorname{det} i \mathbf{B})^{1 / 2} \exp \left(-i \pi \mathbf{r}_{o}^{t} \mathbf{D} \mathbf{B}^{-1} \mathbf{r}_{o}\right) \\
& \times F_{\mathbf{T}}\left(\mathbf{r}_{o}\right) H_{\mathbf{T}}\left(\mathbf{r}_{o}\right),
\end{aligned}
$$

which reduces to the well-known product $(\operatorname{det} i \mathbf{B})^{1 / 2}$ $\times F_{\mathbf{T}}\left(\mathbf{r}_{o}\right) H_{\mathbf{T}}\left(\mathbf{r}_{o}\right)$ when also $\mathbf{D}=\mathbf{0}$, i.e., in the case of a Fourier transformation with scaling and rotation (determined by $\mathbf{B})$, but without an additional phase modulation.

Moreover, by analogy with the alternative representation of the convolution operation via the Fourier domain,

$$
\left(f_{1} * f_{2}\right)(\mathbf{r})=\mathcal{F}^{-1}\left\{\mathcal{F}\left[f_{1}(\cdot)\right](\mathbf{u}) \mathcal{F}\left[f_{2}(\cdot)\right](\mathbf{u})\right\}(\mathbf{r}),
$$

we can introduce the generalized canonical convolution operation as

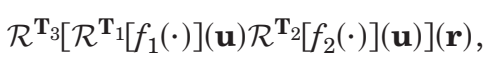

which resembles the common convolution (29) and fractional convolution operations $[6,20,21]$. In particular, if the ray transformation matrices used in Eq. (30) correspond to the ones of the fractional FT, we obtain the generalized fractional convolution, whose applications for shift-variant filtering, encryption, etc., have been proposed [6,20-23]. Since two-dimensional canonical transformations include such operations as rotation, scaling, shearing, fractional FT, etc., the generalized canonical convolution can be helpful for resolving the problem of scale-, rotation-, and shear-invariant (or partially invariant) filtering and pattern recognition.

\section{Scaling Theorem}

To study the CT of a scaled function, we notice that scaling itself belongs to the class of CTs, and we can thus use the additivity property. We then observe that scaling of the input function merely leads to a change of the parameterizing matrix. Indeed, the $\mathbf{T}$-parameterized CT of the scaled function $(\operatorname{det} \mathbf{W})^{1 / 2} f\left(\mathbf{W} \mathbf{r}_{i}\right), \mathcal{R}^{\mathbf{T}}\left[(\operatorname{det} \mathbf{W})^{1 / 2} f\left(\mathbf{W} \mathbf{r}_{i}\right)\right]\left(\mathbf{r}_{o}\right)$, corresponds to the CT of $f\left(\mathbf{r}_{i}\right)$ itself, $\mathcal{R}^{\tilde{\mathbf{T}}}\left[f\left(\mathbf{r}_{i}\right)\right]\left(\mathbf{r}_{o}\right)$, but parameterized by the matrix

$$
\widetilde{\mathbf{T}}=\mathbf{T}\left[\begin{array}{cc}
\mathbf{W}^{-1} & \mathbf{0} \\
\mathbf{0} & \mathbf{W}^{t}
\end{array}\right]
$$


It is useful to find the systems (described by their matrices $\mathbf{T}$ ) and the scaling parameters $\mathbf{W}$ for which scaling of the input function produces only a scaling of the output function:

$$
\mathcal{R}^{\mathbf{T}}\left[(\operatorname{det} \mathbf{W})^{1 / 2} f\left(\mathbf{W} \mathbf{r}_{i}\right)\right]\left(\mathbf{r}_{o}\right)=(\operatorname{det} \mathbf{S})^{1 / 2} \mathcal{R}^{\mathbf{T}}\left[f\left(\mathbf{r}_{i}\right)\right]\left(\mathbf{S r}_{o}\right) .
$$

This condition can be written as

$$
\mathbf{T}\left[\begin{array}{cc}
\mathbf{W}^{-1} & \mathbf{0} \\
\mathbf{0} & \mathbf{W}^{t}
\end{array}\right]=\left[\begin{array}{cc}
\mathbf{S}^{-1} & \mathbf{0} \\
\mathbf{0} & \mathbf{S}^{t}
\end{array}\right] \mathbf{T},
$$

or in detail

$$
\left[\begin{array}{cc}
\mathbf{A W}^{-1} & \mathbf{B} \mathbf{W}^{t} \\
\mathbf{C W}^{-1} & \mathbf{D} \mathbf{W}^{t}
\end{array}\right]=\left[\begin{array}{cc}
\mathbf{S}^{-1} \mathbf{A} & \mathbf{S}^{-1} \mathbf{B} \\
\mathbf{S}^{t} \mathbf{C} & \mathbf{S}^{t} \mathbf{D}
\end{array}\right]
$$

where we will restrict ourselves to separable scaling, i.e., to diagonal matrices $\mathbf{W}$ and $\mathbf{S}$ :

$$
\mathbf{W}=\left[\begin{array}{cc}
w_{x} & 0 \\
0 & w_{y}
\end{array}\right], \quad \mathbf{S}=\left[\begin{array}{cc}
s_{x} & 0 \\
0 & s_{y}
\end{array}\right] .
$$

The condition $\mathbf{A} \mathbf{W}^{-1}=\mathbf{S}^{-1} \mathbf{A}$ leads to the relations $a_{11} w_{x}^{-1}$ $=a_{11} s_{x}^{-1}, \quad a_{12} w_{y}^{-1}=a_{12} s_{x}^{-1}, \quad a_{21} w_{x}^{-1}=a_{21} s_{y}^{-1}, \quad$ and $\quad a_{22} w_{y}^{-1}$ $=a_{22} s_{y}^{-1}$. From $\mathbf{D} \mathbf{W}^{t}=\mathbf{S}^{t} \mathbf{D}$, and since $\mathbf{W}^{t}=\mathbf{W}$ and $\mathbf{S}^{t}=\mathbf{S}$, we conclude that the same relations hold for the coefficients $d_{i j}, i, j=1,2$. From the condition $\mathbf{B} \mathbf{W}^{t}=\mathbf{S}^{-1} \mathbf{B}$ we obtain $b_{11} w_{x}=b_{11} s_{x}^{-1}, \quad b_{12} w_{y}=b_{12} s_{x}^{-1}, \quad b_{21} w_{x}=b_{21} s_{y}^{-1}, \quad$ and $b_{22} w_{y}$ $=b_{22} s_{y}^{-1}$, and the same relations again for $c_{i j}, i, j=1,2$.

If we compare $a_{11} w_{x}^{-1}=a_{11} s_{x}^{-1}$ with $b_{11} w_{x}=b_{11} s_{x}^{-1}$, we conclude that at least one of the coefficients $\left(a_{11}, b_{11}\right)$ should vanish, unless we are dealing with the obvious case $w_{x}=s_{x}=1$, which we will not consider here. Similar observations can be made for all pairs $\left(a_{i j}, b_{i j}\right)$ and $\left(c_{i j}, d_{i j}\right), i, j=1,2$, and we thus conclude that at least eight elements of the $4 \times 4$ ray transformation matrix $\mathbf{T}$ should vanish.

To classify the different possibilities, we consider the relations that may exist between the scaling factors $w_{x}$, $w_{y}, s_{x}$, and $s_{y}$, and get eight different cases:

\begin{tabular}{lll} 
Case Relations & Vanishing Elements \\
\hline
\end{tabular}

$\begin{array}{llllll}1 & w_{x}=w_{y}=s_{x}=s_{y} & b_{11} & b_{12} & b_{21} & b_{22} \\ 2 & w_{x}=w_{y}=s_{x}^{-1}=s_{y}^{-1} & a_{11} & a_{12} & a_{21} & a_{22} \\ 3 & w_{x}=w_{y}^{-1}=s_{x}=s_{y}^{-1} & b_{11} & a_{12} & a_{21} & b_{22} \\ 4 & w_{x}=w_{y}^{-1}=s_{x}^{-1}=s_{y} & a_{11} & b_{12} & b_{21} & a_{22} \\ 5 & w_{x}=w_{y}=s_{x}=s_{y}^{-1} & b_{11} & b_{12} & a_{21} & a_{22} \\ 6 & w_{x}=w_{y}=s_{x}^{-1}=s_{y} & a_{11} & a_{12} & b_{21} & b_{22} \\ 7 & w_{x}=w_{y}^{-1}=s_{x}=s_{y} & b_{11} & a_{12} & b_{21} & a_{22} \\ 8 & w_{x}=w_{y}^{-1}=s_{x}^{-1}=s_{y}^{-1} & a_{11} & b_{12} & a_{21} & b_{22}\end{array}$

Note that if $a_{i j}$ is a vanishing element, then so is $d_{i j}$; if $b_{i j}$ is a vanishing element, then so is $c_{i j}$. Of course, some of the not-necessarily vanishing elements may still vanish, as long as the symplecticity conditions are satisfied.

Case $1\left(w_{x}=w_{y}=s_{x}=s_{y}\right)$ requires all elements $\left(b_{i j}, c_{i j}\right)$ to vanish, and allows nonvanishing elements $\left(a_{i j}, d_{i j}\right)$. The ray transformation matrix takes the form

$$
\mathbf{T}_{1}=\left[\begin{array}{cc}
\mathbf{A}_{1} & \mathbf{0} \\
\mathbf{0} & \mathbf{D}_{1}
\end{array}\right]
$$

where the not-necessarily vanishing elements still have to satisfy four symplecticity conditions, thus reducing the number of free parameters to four; in this particular case we have $\mathbf{D}_{1}=\left(\mathbf{A}_{1}^{t}\right)^{-1}$. Similarly, case $2\left(w_{x}=w_{y}=s_{x}^{-1}=s_{y}^{-1}\right)$ requires all elements $\left(a_{i j}, d_{i j}\right)$ to vanish, and allows nonvanishing elements $\left(b_{i j}, c_{i j}\right)$. We get

$$
\mathbf{T}_{2}=\left[\begin{array}{cc}
\mathbf{0} & \mathbf{B}_{2} \\
\mathbf{C}_{2} & \mathbf{0}
\end{array}\right],
$$

with $\mathbf{C}_{2}=-\left(\mathbf{B}_{2}^{t}\right)^{-1}$. Note that the matrices $\mathbf{T}_{1}$ and $\mathbf{T}_{2}$ differ only by the FT matrix $\mathbf{T}_{f}(\pi / 2, \pi / 2)$ : for example, $\mathbf{T}_{2}$ $=\mathbf{T}_{1} \mathbf{T}_{f}(\pi / 2, \pi / 2)$ corresponds to the identities $\mathbf{B}_{2}=\mathbf{A}_{1}$ and $\mathbf{C}_{2}=-\mathbf{D}_{1}$. Likewise, we can construct the ray transformation matrices for all other cases from the table.

We remark that case 1 corresponds to an ideal imaging operation (with scaling and rotation, such as the skew operation, for example), whereas case 2 corresponds to a FT operation (with scaling and rotation). In particular, $\mathbf{T}_{1}$ can be written as the cascade of a rotator and a magnifier,

$$
\mathbf{T}_{1}=\left[\begin{array}{cc}
\mathbf{S}_{1} \mathbf{X}_{r}(\alpha) & \mathbf{0} \\
\mathbf{0} & \mathbf{S}_{1}^{-1} \mathbf{X}_{r}(\alpha)
\end{array}\right]
$$

with $\mathbf{S}_{1}=\left(\mathbf{A}_{1} \mathbf{A}_{1}^{t}\right)^{1 / 2}$ a symmetric matrix; see also the Iwasawa decomposition, Eqs. (3)-(6). Similarly, for case 2 we have

$$
\mathbf{T}_{2}=\left[\begin{array}{cc}
\mathbf{0} & \mathbf{S}_{2} \mathbf{X}_{r}(\alpha) \\
-\mathbf{S}_{2}^{-1} \mathbf{X}_{r}(\alpha) & \mathbf{0}
\end{array}\right],
$$

with $\mathbf{S}_{2}=\left(\mathbf{B}_{2} \mathbf{B}_{2}^{t}\right)^{1 / 2}$. Note that we have indeed exactly four free parameters: the rotating angle $\alpha$ and the three elements that determine the symmetric $2 \times 2$ matrix $\mathbf{S}$.

Case 3 has as a special case the gyrator; see Eq. (18). In general, case 3 is a scaled version of a gyrator (with gyrating angle $\vartheta$ ) scaled with a diagonal scaling matrix $\mathbf{S}_{3}$ (and thus described by two parameters), followed by a very special lens with an antidiagonal symmetric lens matrix $\mathbf{G}_{3}$ (described by one parameter):

$$
\mathbf{S}_{3}=\left[\begin{array}{cc}
s_{11} & 0 \\
0 & s_{22}
\end{array}\right], \quad \mathbf{G}_{3}=\left[\begin{array}{ll}
0 & g \\
g & 0
\end{array}\right]
$$

The matrix $\mathbf{T}_{3}$ can thus be decomposed as

$$
\begin{aligned}
& \mathbf{A}_{3}=\mathbf{S}_{3} \mathbf{X}_{g}(\vartheta) \\
& \mathbf{B}_{3}=\mathbf{S}_{3} \mathbf{Y}_{g}(\vartheta), \\
& \mathbf{C}_{3}=-\mathbf{G}_{3} \mathbf{S}_{3} \mathbf{X}_{g}(\vartheta)-\mathbf{S}_{3}^{-1} \mathbf{Y}_{g}(\vartheta), \\
& \mathbf{D}_{3}=-\mathbf{G}_{3} \mathbf{S}_{3} \mathbf{Y}_{g}(\vartheta)+\mathbf{S}_{3}^{-1} \mathbf{X}_{g}(\vartheta)
\end{aligned}
$$


Like cases 1 and 2 [with $\mathbf{T}_{2}=\mathbf{T}_{1} \mathbf{T}_{f}(\pi / 2, \pi / 2)$ ], cases 3 and 4 [with $\mathbf{T}_{4}=\mathbf{T}_{3} \mathbf{T}_{f}(\pi / 2, \pi / 2)$ ] differ only by an additional FT operation: for the matrix $\mathbf{T}_{4}$ we thus have

$$
\begin{aligned}
& \mathbf{A}_{4}=-\mathbf{S}_{4} \mathbf{Y}_{g}(\vartheta) \\
& \mathbf{B}_{4}=\mathbf{S}_{4} \mathbf{X}_{g}(\vartheta) \\
& \mathbf{C}_{4}=\mathbf{G}_{4} \mathbf{S}_{4} \mathbf{Y}_{g}(\vartheta)-\mathbf{S}_{4}^{-1} \mathbf{X}_{g}(\vartheta), \\
& \mathbf{D}_{4}=-\mathbf{G}_{4} \mathbf{S}_{4} \mathbf{X}_{g}(\vartheta)-\mathbf{S}_{4}^{-1} \mathbf{Y}_{g}(\vartheta)
\end{aligned}
$$

where $\mathbf{S}_{4}$ and $\mathbf{G}_{4}$ have the same structure as $\mathbf{S}_{3}$ and $\mathbf{G}_{3}$, respectively. Note that we have again exactly four free parameters: the gyrating angle $\vartheta$, the parameter $g$ that determines the antidiagonal symmetric $2 \times 2$ matrix $\mathbf{G}$, and the two elements that determine the diagonal $2 \times 2$ matrix $\mathbf{S}$.

We finally note that for the remaining cases $5,6,7$, and 8, all submatrices $\mathbf{A}, \mathbf{B}, \mathbf{C}$, and $\mathbf{D}$ are singular, see $\mathbf{T}_{5}$ as an example,

$$
\mathbf{T}_{5}=\left[\begin{array}{cccc}
a_{11} & a_{12} & 0 & 0 \\
0 & 0 & b_{21} & b_{22} \\
0 & 0 & d_{11} & d_{12} \\
c_{21} & c_{22} & 0 & 0
\end{array}\right],
$$

and cases 5 and 6 differ only by a FT operation again, as do cases 7 and 8. Moreover, from the Iwasawa decomposition we learn that an antidiagonal symmetric lens matrix $\mathbf{G}$ appears again in cases 5 and 6 , while cases 7 and 8 do not require such a lens (since $\mathbf{C A}^{t}+\mathbf{D B} \mathbf{B}^{t}=\mathbf{0}$ ).

D. Derivation by $\left(\partial^{m} / \partial x^{m}, \partial^{n} / \partial y^{n}\right)$ : Multiplication by $\left(x^{k}, y^{l}\right)$

With $\nabla=(\partial / \partial x, \partial / \partial y)^{t}$ and $\operatorname{det} \mathbf{B} \neq 0$, the gradient of the CT can be written

$$
\begin{aligned}
\nabla_{o} f_{o}\left(\mathbf{r}_{o}\right) & =\nabla_{o}\left\{\mathcal{R}^{\mathbf{T}}\left[f_{i}\left(\mathbf{r}_{i}\right)\right]\left(\mathbf{r}_{o}\right)\right\} \\
& =i 2 \pi\left(\mathbf{B}^{t}\right)^{-1}\left\{\mathbf{D}^{t} \mathbf{r}_{o} f_{o}\left(\mathbf{r}_{o}\right)-\mathcal{R}^{\mathbf{T}}\left[\mathbf{r}_{i} f_{i}\left(\mathbf{r}_{i}\right)\right]\left(\mathbf{r}_{o}\right)\right\},
\end{aligned}
$$

where we have used the symplecticity condition $\mathbf{B}^{t} \mathbf{D}$ $=\mathbf{D}^{t} \mathbf{B}$. We thus obtain

$$
\mathcal{R}^{\mathbf{T}}\left[\mathbf{r}_{i} f_{i}\left(\mathbf{r}_{i}\right)\right]\left(\mathbf{r}_{o}\right)=\left\{\mathbf{D}^{t} \mathbf{r}_{o}-\frac{\mathbf{B}^{t}}{i 2 \pi} \nabla_{o}\right\} f_{o}\left(\mathbf{r}_{o}\right) .
$$

To find an expression for $\mathcal{R}^{\mathbf{T}}\left[\nabla_{i} f_{i}\left(\mathbf{r}_{i}\right)\right]\left(\mathbf{r}_{o}\right)$, we apply the inverse CT to Eq. (42) yielding

$$
\mathcal{R}^{\mathbf{T}^{-1}}\left[\nabla_{o} f_{o}\left(\mathbf{r}_{o}\right)\right]\left(\mathbf{r}_{i}\right)=i 2 \pi\left(\mathbf{B}^{t}\right)^{-1}\left\{\mathbf{D}^{t} \mathcal{R}^{\mathbf{T}^{-1}}\left[\mathbf{r}_{o} f_{o}\left(\mathbf{r}_{o}\right)\right]\left(\mathbf{r}_{i}\right)-\mathbf{r}_{i} f_{i}\left(\mathbf{r}_{i}\right)\right\},
$$

and with the changes $\mathbf{T} \rightarrow \mathbf{T}^{-1}, \mathbf{B} \rightarrow-\mathbf{B}^{t}, \mathbf{D} \rightarrow \mathbf{A}^{t}, o \rightarrow i$, and $i \rightarrow o$, we get

$$
\mathcal{R}^{\mathbf{T}}\left[\nabla_{i} f_{i}\left(\mathbf{r}_{i}\right)\right]\left(\mathbf{r}_{o}\right)=i 2 \pi(-\mathbf{B})^{-1}\left\{\mathbf{A} \mathcal{R}^{\mathbf{T}}\left[\mathbf{r}_{i} f_{i}\left(\mathbf{r}_{i}\right)\right]\left(\mathbf{r}_{o}\right)-\mathbf{r}_{o} f_{o}\left(\mathbf{r}_{o}\right)\right\} .
$$

After substituting from Eq. (43) and using the symplecticity conditions, we thus get

$$
\mathcal{R}^{\mathbf{T}}\left[\nabla_{i} f_{i}\left(\mathbf{r}_{i}\right)\right]\left(\mathbf{r}_{o}\right)=\left(-i 2 \pi \mathbf{C}^{t} \mathbf{r}_{o}+\mathbf{A}^{t} \nabla_{o}\right) f_{o}\left(\mathbf{r}_{o}\right) .
$$

We may combine Eqs. (43) and (44), and write

$$
\mathcal{R}^{\mathbf{T}}\left[\mathbf{z}_{i} f_{i}\left(\mathbf{r}_{i}\right)\right]\left(\mathbf{r}_{o}\right)=\mathbf{z}_{o} f_{o}\left(\mathbf{r}_{o}\right),
$$

where we have introduced the symbolic vectors

$$
\mathbf{z}_{i}=\left(\begin{array}{c}
i 2 \pi \mathbf{r}_{i} \\
\nabla_{i}
\end{array}\right), \quad \mathbf{z}_{o}=\mathbf{T}^{-1}\left(\begin{array}{c}
i 2 \pi \mathbf{r}_{o} \\
\nabla_{o}
\end{array}\right)
$$

Since each row in the vector equation (45) operates independently of the others, we may take any operator to an arbitrary, nonnegative, integer power. With $\mathbf{z}^{\{k l m n\}}$ defined as $\mathbf{z}^{\{k l m n\}}=\left(z_{1}^{k}, z_{2}^{l}, z_{3}^{m}, z_{4}^{n}\right)^{t}(k, l, m, n=0,1, \ldots)$, Eq. (45) can then be generalized to

$$
\mathcal{R}^{\mathbf{T}}\left[\mathbf{z}_{i}^{\{k l m n\}} f_{i}\left(\mathbf{r}_{i}\right)\right]\left(\mathbf{r}_{o}\right)=\mathbf{z}_{o}^{\{k l m n\}} f_{o}\left(\mathbf{r}_{o}\right) .
$$

In particular, if $\mathbf{A}=\mathbf{0}$ we obtain a relation that resembles the well-known formula for the FT,

$$
\mathcal{R}^{\mathbf{T}}\left[\nabla_{i}^{\{m n\}} f_{i}\left(\mathbf{r}_{i}\right)\right]\left(\mathbf{r}_{o}\right)=\left(-i 2 \pi \mathbf{C}^{t} \mathbf{r}_{o}\right)^{\{m n\}} \mathcal{R}^{\mathbf{T}}\left[f_{i}(\cdot)\right]\left(\mathbf{r}_{o}\right),
$$

while for $\mathbf{C}=\mathbf{0}$ we obtain a formula similar to one known for the convolution-type transformation,

$$
\mathcal{R}^{\mathbf{T}}\left[\nabla_{i}^{\{m n\}} f_{i}\left(\mathbf{r}_{i}\right)\right]\left(\mathbf{r}_{o}\right)=\left(\mathbf{A}^{t} \nabla_{o}\right)^{\{m n\}} \mathcal{R}^{\mathbf{T}}\left[f_{i}\left(\mathbf{r}_{i}\right)\right]\left(\mathbf{r}_{o}\right) .
$$

\section{CANONICAL TRANSFORMS OF SELECTED FUNCTIONS}

\section{A. Exponentials with a Quadratic Argument}

For a limited number of functions, an analytical expression of their CTs can be found. Among them we mention the function

$$
f_{i}(\mathbf{r})=\exp \left(i 2 \pi \mathbf{k}_{i}^{t} \mathbf{r}-\pi \mathbf{r}^{t} \mathbf{L}_{i} \mathbf{r}\right),
$$

which describes as particular cases a plane wave, a Gaussian beam, an elliptical wave, and a hyperbolic wave; note that we may choose the matrix $\mathbf{L}_{i}$ to be symmetric and that we require that its real part be nonnegative definite. Following the calculations done in [24] it is easy to prove that the $\mathrm{CT}$ of the function (50) takes the form

$$
\begin{aligned}
f_{o}(\mathbf{r})= & \mathcal{R}^{\mathbf{T}}\left[f_{i}(\cdot)\right](\mathbf{r})=\left[\operatorname{det}\left(\mathbf{A}+i \mathbf{B} \mathbf{L}_{i}\right)\right]^{-1 / 2} \\
& \times \exp \left[-i \pi \mathbf{k}_{i}^{t}\left(\mathbf{A}+i \mathbf{B} \mathbf{L}_{i}\right)^{-1} \mathbf{B k}_{i}+i 2 \pi \mathbf{k}_{o}^{t} \mathbf{r}-\pi \mathbf{r}^{t} \mathbf{L}_{o} \mathbf{r}\right],
\end{aligned}
$$

where

$$
\begin{gathered}
\mathbf{k}_{o}^{t}=\mathbf{k}_{i}^{t}\left(\mathbf{A}+i \mathbf{B} \mathbf{L}_{i}\right)^{-1}, \\
i \mathbf{L}_{o}=\left(\mathbf{C}+i \mathbf{D} \mathbf{L}_{i}\right)\left(\mathbf{A}+i \mathbf{B L}_{i}\right)^{-1} .
\end{gathered}
$$

For $\mathbf{L}_{i}=\mathbf{0}$ and a real-valued wave vector $2 \pi \mathbf{k}_{i}$, the input function $f_{i}(\mathbf{r})=\exp \left(i 2 \pi \mathbf{k}_{i}^{t} \mathbf{r}\right)$ corresponds to a plane wave, and its $\mathrm{CT}$ is given by

$$
\begin{aligned}
f_{o}(\mathbf{r})= & (\operatorname{det} \mathbf{A})^{-1 / 2} \exp \left(-i \pi \mathbf{k}_{i}^{t} \mathbf{A}^{-1} \mathbf{B} \mathbf{k}_{i}+i 2 \pi \mathbf{k}_{i}^{t} \mathbf{A}^{-1} \mathbf{r}\right. \\
& \left.+i \pi \mathbf{r}^{t} \mathbf{C A}^{-1} \mathbf{r}\right) .
\end{aligned}
$$

In particular, this implies that a plane wave remains a plane wave under the $\mathrm{CT}$ only if $\mathbf{C}=\mathbf{0}$, which again stresses the interpretation of this case as a generalized Fresnel transformation. 
The case $\mathbf{k}_{i}=\mathbf{0}$, and thus $f_{i}(\mathbf{r})=\exp \left(-\pi \mathbf{r}^{t} \mathbf{L}_{i} \mathbf{r}\right)$, deserves some separate attention as well. It contains as special cases: (i) a Gaussian beam $\exp \left[-\pi\left(l_{11} x^{2}+2 l_{12} x y+l_{22} y^{2}\right)\right]$ (when $\mathbf{L}_{i}$ is real and positive definite), and (ii) an elliptical wave $\exp \left[i \pi\left(h_{11} x^{2}+2 h_{12} x y+h_{22} y^{2}\right)\right]$ (when $\mathbf{L}_{i}=-i \mathbf{H}$ is imaginary), among which we have the particular cases of an elliptical wave $\exp \left[i \pi\left(h_{11} x^{2}+h_{22} y^{2}\right)\right]$ aligned along the coordinate axes, a spherical wave $\exp \left[i \pi h\left(x^{2}+y^{2}\right)\right]$, and a hyperbolic wave $\exp [i 2 \pi h x y]$, in which three cases $\mathbf{H}$ is a diagonal matrix, a scalar matrix, and an antidiagonal matrix, respectively. For $\mathbf{k}_{i}=\mathbf{0}$, the CT takes the simple form

$$
f_{o}(\mathbf{r})=\left[\operatorname{det}\left(\mathbf{A}+i \mathbf{B} \mathbf{L}_{i}\right)\right]^{-1 / 2} \exp \left(-\pi \mathbf{r}^{t} \mathbf{L}_{o} \mathbf{r}\right)
$$

and the relation between $\mathbf{L}_{o}$ and $\mathbf{L}_{i}$ is given by the wellknown ABCD law of Eq. (53). Note that if $\mathbf{L}_{i}=-i \mathbf{H}_{i}$ is imaginary, then $\mathbf{L}_{o}=-i \mathbf{H}_{o}$ is imaginary, too, and the ABCD law takes the common form

$$
\mathbf{H}_{o}=\left(\mathbf{C}+\mathbf{D H}_{i}\right)\left(\mathbf{A}+\mathbf{B H}_{i}\right)^{-1},
$$

in which only real-valued parameters are involved.

On the basis of the ABCD law of Eq. (56), $\mathbf{H}_{o}(\mathbf{A}$ $\left.+\mathbf{B H}_{i}\right)=\mathbf{C}+\mathbf{D H}_{i}$, we can find systems that, for example, transform an aligned elliptical wave into a hyperbolic one, or maintain the aligned elliptical or hyperbolic structure during the transformation. In particular, an aligned elliptical wave (with diagonal matrix $\mathbf{H}_{i}$ ) remains aligned (with diagonal matrix $\mathbf{H}_{o}$ ) after propagation through a first-order optical system, if all block matrices are diagonal; this means that the CT is separable. Analogously, a hyperbolic wave (with antidiagonal matrix $\mathbf{H}_{i}$ ) remains hyperbolic (with antidiagonal matrix $\mathbf{H}_{o}$ ) if $\mathbf{A}$ and $\mathbf{D}$ are diagonal and $\mathbf{B}$ and $\mathbf{C}$ are antidiagonal; an example is the CT performed by a gyrator.

It is easy to see that the CT of the Dirac $\delta$ function is given by

$$
\begin{aligned}
\mathcal{R}^{\mathbf{T}}\left[\delta\left(\mathbf{r}_{i}-\mathbf{v}\right)\right]\left(\mathbf{r}_{o}\right)= & (\operatorname{det} i \mathbf{B})^{-1 / 2} \exp \left[i \pi \left(\mathbf{v}^{t} \mathbf{B}^{-1} \mathbf{A} \mathbf{v}\right.\right. \\
& \left.\left.-2 \mathbf{v}^{t} \mathbf{B}^{-1} \mathbf{r}_{o}+\mathbf{r}_{o}^{t} \mathbf{D} \mathbf{B}^{-1} \mathbf{r}_{o}\right)\right]
\end{aligned}
$$

The resulting elliptical wave becomes an aligned elliptical wave (also called a chirp signal) if $\mathbf{D B}^{-1}$ is a diagonal matrix, which is the case for the fractional FT, and it becomes a hyperbolic wave if $\mathbf{D B}^{-1}$ is antidiagonal, which is the case for a gyrator operation. This implies that we can use fractional FT and gyrator systems for the localization of chirp signals and hyperbolic waves, respectively.

\section{B. Periodic Functions: Talbot Imaging}

Let us consider the propagation of a periodic field with periods $p_{x}$ and $p_{y}$ with respect to the $x$ and $y$ coordinates through a first-order optical system. When the function $f_{i}(\mathbf{r})$ is represented as a superposition of plane waves,

$$
f_{i}(\mathbf{r})=\sum_{m, n=-\infty}^{\infty} a_{m n} \exp \left(i 2 \pi \mathbf{k}_{m n}^{t} \mathbf{r}\right)
$$

with $\mathbf{k}_{m n}^{t}=\left(m / p_{x}, n / p_{y}\right)$, then the CT of this function can be written in the form

$$
\begin{aligned}
f_{o}(\mathbf{r})= & (\operatorname{det} \mathbf{A})^{-1 / 2} \sum_{m, n=-\infty}^{\infty} a_{m n} \exp \left(-i \pi \mathbf{k}_{m n}^{t} \mathbf{A}^{-1} \mathbf{B k}_{m n}\right. \\
& \left.+i 2 \pi \mathbf{k}_{m n}^{t} \mathbf{A}^{-1} \mathbf{r}+i \pi \mathbf{r}^{t} \mathbf{C A}^{-1} \mathbf{r}\right)
\end{aligned}
$$

If $\mathbf{k}_{m n}^{t} \mathbf{A}^{-1} \mathbf{B k}_{m n}$ is an even integer,

$$
\mathbf{k}_{m n}^{t} \mathbf{A}^{-1} \mathbf{B} \mathbf{k}_{m n}=2 \ell
$$

we get

$$
f_{o}(\mathbf{r})=(\operatorname{det} \mathbf{A})^{-1 / 2} \exp \left(i \pi \mathbf{r}^{t} \mathbf{C} \mathbf{A}^{-1} \mathbf{r}\right) f_{i}\left(\mathbf{A}^{-1} \mathbf{r}\right)
$$

which corresponds to generalized Talbot imaging, including a rotation and a scaling of the coordinates described by the matrix $\mathbf{A}^{-1}$ and a phase modulation described by the matrix product $\mathbf{C A}^{-1}$. We stress again the similarity with the Fresnel transformation for $\mathbf{C}=\mathbf{0}$, in which case only an affine transformation of the input function remains.

Let us study the generalized Talbot condition (60), which reads in detail

$$
\frac{q_{11}}{p_{x}^{2}} m^{2}+2 \frac{q_{12}}{p_{x} p_{y}} m n+\frac{q_{22}}{p_{y}^{2}} n^{2}=2 \ell,
$$

where $q_{11}, q_{12}$, and $q_{22}$ are the elements of the symmetric matrix $\mathbf{Q}=\mathbf{A}^{-1} \mathbf{B}$. Note that this equation has to hold for any integers $m$ and $n$, and therefore we have three conditions: $q_{11} / p_{x}^{2}, 2 q_{12} / p_{x} p_{y}$, and $q_{22} / p_{y}^{2}$ should be even integers.

In the case of an orthosymplectic transformation, which can be represented [12] as $\mathbf{T}_{r}(\beta) \mathbf{T}_{f}\left(\gamma_{x}, \gamma_{y}\right) \mathbf{T}_{r}(\alpha)$, the matrix $\mathbf{Q}$ is given by

$$
\left[\begin{array}{cc}
\cos ^{2} \alpha \tan \gamma_{x}+\sin ^{2} \alpha \tan \gamma_{y} & \cos \alpha \sin \alpha\left(\tan \gamma_{x}-\tan \gamma_{y}\right) \\
\cos \alpha \sin \alpha\left(\tan \gamma_{x}-\tan \gamma_{y}\right) & \sin ^{2} \alpha \tan \gamma_{x}+\cos ^{2} \alpha \tan \gamma_{y}
\end{array}\right] .
$$

For orthosymplectic transformations, the Talbot condition depends on only three parameters of the ray transformation matrix, since the fourth one (the angle $\beta$ ) is related to the final rotation, and can be written as

$$
\begin{gathered}
\left(\frac{m}{p_{x}}\right)^{2}\left(\cos ^{2} \alpha \tan \gamma_{x}+\sin ^{2} \alpha \tan \gamma_{y}\right)+\frac{m m}{p_{x} p_{y}} \sin 2 \alpha\left(\tan \gamma_{x}\right. \\
\left.-\tan \gamma_{y}\right)+\left(\frac{n}{p_{y}}\right)^{2}\left(\sin ^{2} \alpha \tan \gamma_{x}+\cos ^{2} \alpha \tan \gamma_{y}\right)=2 \ell .
\end{gathered}
$$

In the case $\gamma_{x}=\gamma_{y}=\gamma$, this reduces to

$$
\tan \gamma\left[\left(\frac{m}{p_{x}}\right)^{2}+\left(\frac{n}{p_{y}}\right)^{2}\right]=2 \ell
$$

and in the case $\gamma_{x}=-\gamma_{y}=\gamma$ to

$$
\cos 2 \alpha \tan \gamma\left[\left(\frac{m}{p_{x}}\right)^{2}-\left(\frac{n}{p_{y}}\right)^{2}\right]+\frac{m n}{p_{x} p_{y}} 2 \sin 2 \alpha \tan \gamma=2 \ell .
$$

We recall that orthosymplectic systems include the separable FT (for $\alpha=\beta=0$ ) and the gyrator (for $\alpha=\pi / 4, \gamma_{x}=$ $\left.-\gamma_{y}=\vartheta\right)$; their matrices $\mathbf{Q}_{f}\left(\gamma_{x}, \gamma_{y}\right)$ and $\mathbf{Q}_{g}(\vartheta)$ thus take the forms 


$$
\mathbf{Q}_{f}=\left[\begin{array}{cc}
\tan \gamma_{x} & 0 \\
0 & \tan \gamma_{y}
\end{array}\right], \quad \mathbf{Q}_{g}=\left[\begin{array}{cc}
0 & \tan \vartheta \\
\tan \vartheta & 0
\end{array}\right] .
$$

In the case of a separable fractional FT, the Talbot condition reads $\left(m / p_{x}\right)^{2} \tan \gamma_{x}+\left(n / p_{y}\right)^{2} \tan \gamma_{y}=2 \ell$ and Talbot imaging occurs for the fractional angles $\tan \gamma_{x, y}=2 \ell p_{x, y}^{2}$, while for the gyrator operation we obtain $\tan \vartheta=l p_{x} p_{y}$.

\section{CONCLUSIONS}

We have derived the main theorems for the twodimensional canonical integral transformation. Some particular CTs such as fractional FT, image scaling, rotation, and Fresnel transformation have already been applied widely in optics and signal processing. Moreover, the gyrator operation has been found useful for the description of mode converters. The discussed properties of the CT open new perspectives for its application in information processing and in particular for its implementation by first-order optical systems. Signal processing in a canonical domain offers many degrees of freedom and can therefore be better adapted to a specific task.

\section{ACKNOWLEDGMENTS}

T. Alieva acknowledges the Spanish Ministry of Education and Science for financial support (project TEC 200502180/MIC). M. J. Bastiaans appreciates the hospitality of Universidad Complutense de Madrid.

\section{REFERENCES}

1. M. Moshinsky and C. Quesne, "Linear canonical transformations and their unitary representations," J. Math. Phys. 12, 1772-1780 (1971).

2. K. B. Wolf, Integral Transforms in Science and Engineering (Plenum, 1979).

3. M. Nazarathy and J. Shamir, "First-order optics - a canonical operator representation: lossless systems," J. Opt. Soc. Am. 72, 356-364 (1982).

4. S. C. Pei and J. J. Ding, "Eigenfunctions of linear canonical transform," IEEE Trans. Signal Process. 50, 11-26 (2002).

5. H. M. Ozaktas, Z. Zalevsky, and M. A. Kutay, The Fractional Fourier Transform with Applications in Optics and Signal Processing (Wiley, 2001).

6. T. Alieva, M. J. Bastiaans, and M. L. Calvo, "Fractional transforms in optical information processing," EURASIP
(European Association for Signal and Image Processing) J. Appl. Signal Process. 2005: 10, 1498-1519 (2005).

7. L. Allen, M. W. Beijersbergen, R. J. C. Spreeuw, and J. P. Woerdman, "Orbital angular momentum of light and the transformation of Laguerre Gaussian laser modes," Phys. Rev. A 45, 8185-8189 (1992).

8. E. G. Abramochkin and V. G. Volostnikov, "Generalized Gaussian beams,” J. Opt. A, Pure Appl. Opt. 6, S157-S161 (2004).

9. R. Simon and K. B. Wolf, "Fractional Fourier transforms in two dimensions," J. Opt. Soc. Am. A 17, 2368-2381 (2000).

10. K. B. Wolf, Geometric Optics on Phase Space (Springer, 2004).

11. S. A. Collins, Jr., "Lens-system diffraction integral written in terms of matrix optics," J. Opt. Soc. Am. 60, 1168-1177 (1970).

12. T. Alieva and M. J. Bastiaans, "Alternative representation of the linear canonical integral transform," Opt. Lett. 30, 3302-3304 (2005).

13. J. A. Rodrigo, T. Alieva, and M. L. Calvo, "Optical system design for ortho-symplectic transformations in phase space," J. Opt. Soc. Am. A 23, 2494-2500 (2006).

14. A. W. Lohmann, "Image rotation, Wigner rotation, and the fractional Fourier transform,” J. Opt. Soc. Am. A 10, 2181-2186 (1993).

15. M. J. Bastiaans and T. Alieva, "Synthesis of an arbitrary $A B C D$ system with fixed lens positions," Opt. Lett. 31, 2414-2416 (2006).

16. M. J. Bastiaans and T. Alieva, "Classification of lossless first-order optical systems and the linear canonical transformation," J. Opt. Soc. Am. A 24, 1053-1062 (2007).

17. T. Alieva and M. J. Bastiaans, "Mode mapping in paraxial lossless optics," Opt. Lett. 30, 1461-1463 (2005).

18. M. J. Bastiaans and T. Alieva, "First-order optical systems with unimodular eigenvalues," J. Opt. Soc. Am. A 23, 1875-1883 (2006).

19. M. J. Bastiaans and T. Alieva, "First-order optical systems with real eigenvalues," Opt. Commun. 272, 52-55 (2007).

20. A. W. Lohmann, Z. Zalevsky, and D. Mendlovic, "Synthesis of pattern recognition filters for fractional Fourier processing," Opt. Commun. 128, 199-204 (1996).

21. O. Akay and G. F. Boudreaux-Bartels, "Fractional convolution and correlation via operator methods and an application to detection of linear FM signals," IEEE Trans. Signal Process. 49, 979-993 (2001).

22. J. García, D. Mendlovic, Z. Zalevsky, and A. W. Lohmann, "Space-variant simultaneous detection of several objects by the use of multiple anamorphic fractional-Fourier transform filters," Appl. Opt. 35, 3945-3952 (1996).

23. B. Zhu and S. Liu, "Optical image encryption based on the generalized fractional convolution operation," Opt. Commun. 195, 371-381 (2001).

24. M. J. Bastiaans and T. Alieva, "Propagation law for the generating function of Hermite-Gaussian-type modes in first-order optical systems," Opt. Express 13, 1107-1112 (2005). 